\title{
Surgical Treatment for Hypertrophic Obstructive Cardiomyopathy with Concomitant Mitral Valve Abnormalities: A Cohort of 26 Cases
}

\author{
Bang-rong Song ${ }^{1}$, Yan-long Ren ${ }^{2}$, Hong-jia Zhang ${ }^{1}$ \\ ${ }^{1}$ Department of Cardiac Surgery, Beijing Anzhen Hospital, Capital Medical University, Beijing 100029, China; ${ }^{2}$ Department of \\ Cardiology, Beijing Anzhen Hospital, Capital Medical University, Beijing 100029, China.
}

\section{ABSTRACT}

Background: We sought to analyze the pathological characteristics of hypertrophic obstructive cardiomyopathy (HOCM) with concomitant mitral valve abnormalities and to discuss the surgical treatment strategies.

Methods: The clinical data of $26 \mathrm{HOCM}$ patients treated from January 2014 to March 2016 were retrospectively analyzed. There were 19 males and 7 females with a mean age of $47 \pm 16$ years (range, 10-70 years). Echocardiography showed HOCM, systolic anterior motion of the mitral apparatus, and concomitant mitral regurgitation. Modified Morrow procedure with expanded resection area was performed in 21 patients. Concomitant mitral valvuloplasty was performed in 4 patients, coronary artery bypass grafting was performed in one patient, and aortic valve replacement was performed in one patient. Echocardiography was performed intraoperatively at postoperative 1 week and at postoperative 1 year to evaluate the left ventricular obstruction and the mitral regurgitation.

Results: The left ventricular outflow tract gradient, left ventricular outflow tract velocity, septal thickness, and mitral regurgitation area decreased significantly at postoperative 1 week and 1 year in comparison with the baseline (all $P<.001$ ). The postoperative mitral regurgitation and systolic anterior motion of the mitral apparatus were completely abolished or significantly relieved. Only one patient had moderate mitral regurgitation of $7 \mathrm{~cm}^{2}$ after the surgery. At postoperative 1 year, all patients were asymptomatic, and the quality of life was significantly improved. The New York Heart Association (NYHA) class was I-II. Echocardiography showed good anatomy and function of the mitral valve.

Conclusions: Concomitant mitral valve abnormality is not uncommon in HOCM. Septal myectomy can adequately expand the left ventricular outflow tract and abolish mitral regurgitation and systolic anterior motion of the mitral apparatus. Concomitant mitral valvuloplasty is indicated for severe congenital abnormalities or secondary lesions of the mitral valve, and the outcomes are satisfactory.

Received March 30, 2018; received in revised form September 26, 2018; accepted September 28, 2018.

Correspondence: Dr. Hong-jia Zhang, Department of Cardiac Surgery, Beijing Anzhen Hospital, Capital Medical University, No.2, Anzhen Road, Beijing 100029, China; +86-10-64412431; fax: +86-10-64412431 (e-mail: bjzhang_med@126.com).

\section{INTRODUCTION}

Hypertrophic obstructive cardiomyopathy (HOCM) is an autosomal dominant genetic disease characterized by hypertrophied septum and left ventricle, which result in increased myocardial contractility and decreased diastolic compliance. Septal myectomy can effectively reduce obstruction of the left ventricular outflow tract and has become the standard treatment for patients with HOCM [Maron 2002; Maron 2003; Elliott 2004; Gersh 2011].
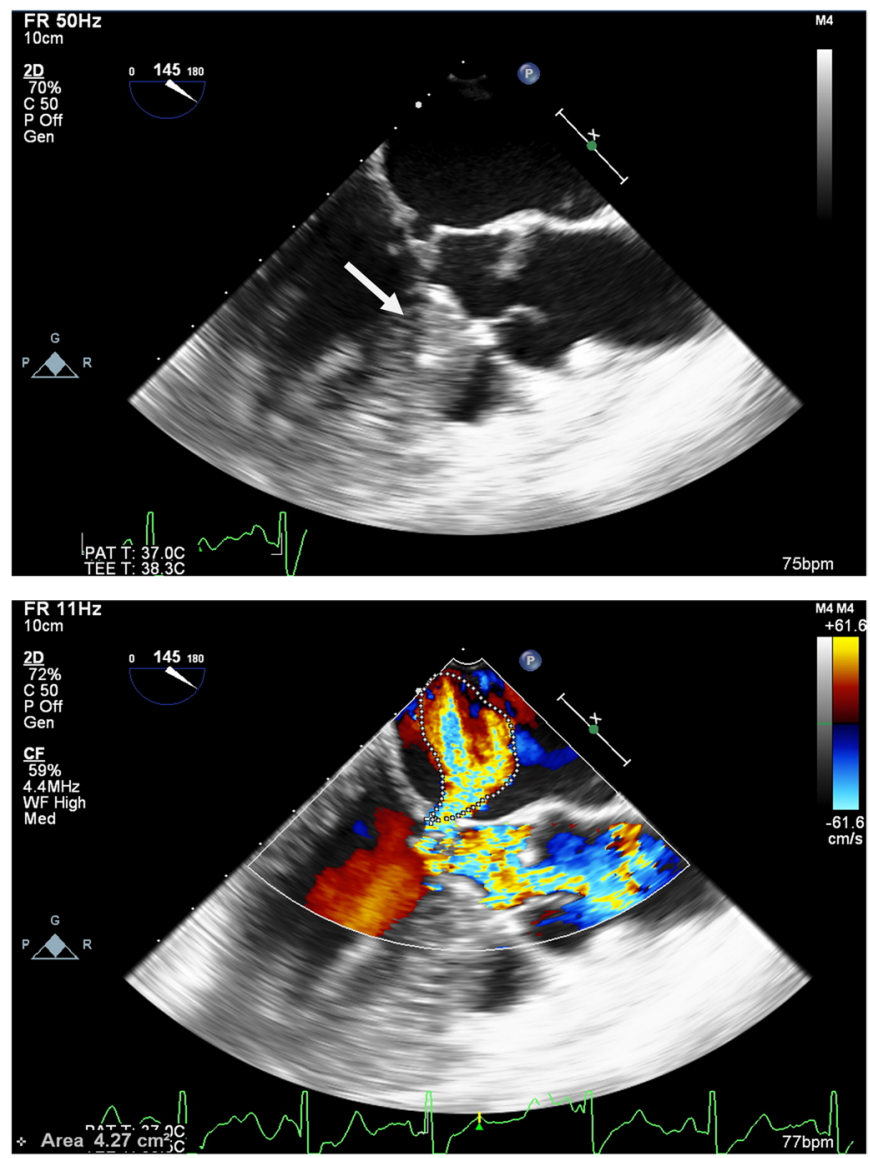

Figure 1. Preoperative echocardiographic images of the interventricular septum in a patient. The 2D echocardiography (upper panel) showed the hypertrophied interventricular septum (white arrow), which caused mitral regurgitation. The Doppler ultrasound (lower panel) showed the significant mitral regurgitation (dotted area). 

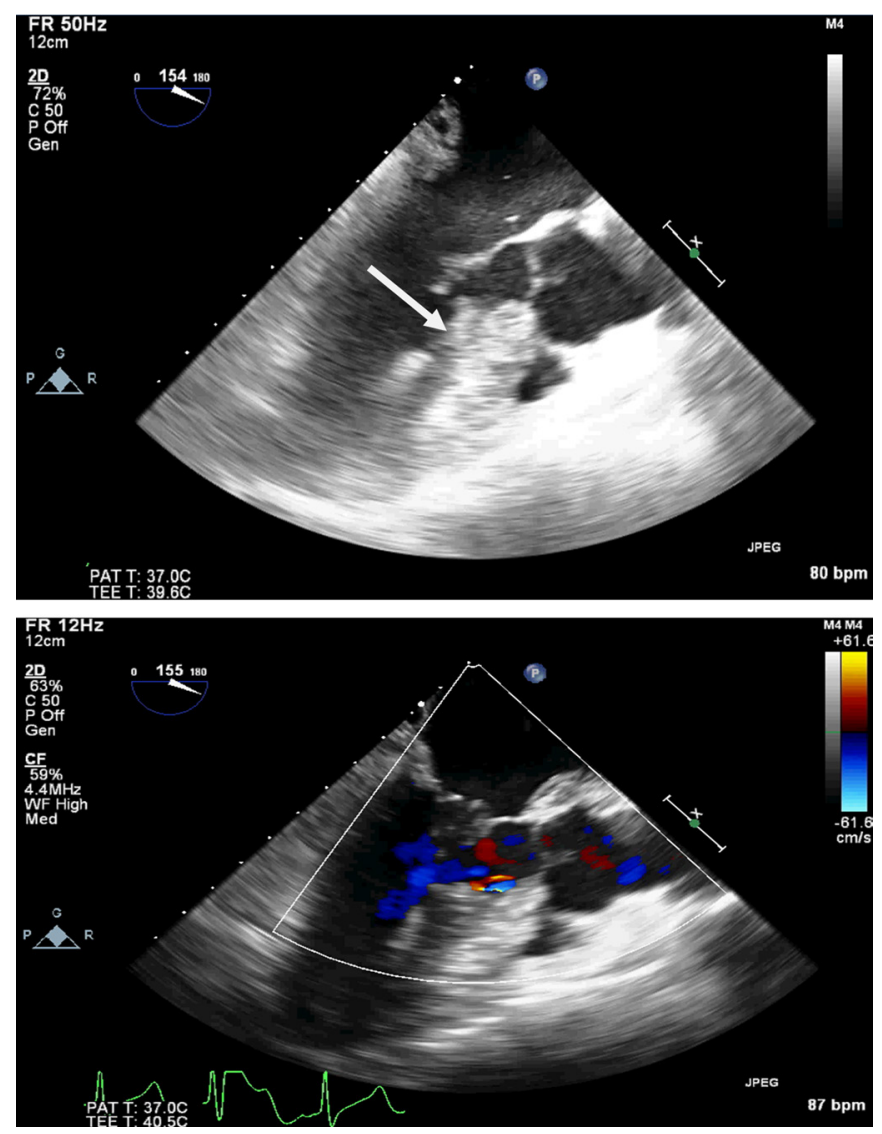

Figure 2. Postoperative echocardiographic images of the interventricular septum in a patient. The 2D echocardiography (upper panel) showed the interventricular septum (while arrow) after resection. The Doppler ultrasound (lower panel) showed that the marked mitral regurgitation had disappeared.

Systolic anterior motion of the mitral apparatus is a characteristic pathological change in HOCM. In addition, patients with HOCM often have concomitant mitral regurgitation. Mitral regurgitation is caused by mitral valve lesions in only $4 \%$ to $14 \%$ of the HOCM patients, such as mitral valve inflammation, abnormalities of the papillary muscles or chorda, mucoid degenerations, and retrograde degenerations. In most conditions, mitral regurgitation is closely associated with the basic pathological changes of HOCM. Mitral valve replacement can abolish the systolic anterior motion of the mitral apparatus but put patients at risks of artificial valve infection and anticoagulation complications.

There is no accepted treatment method for HOCM with concomitant mitral regurgitation. Here we report our experiences with 26 HOCM patients treated with modified Morrow septal myectomy.

\section{MATERIALS AND METHODS}

From January 2014 to March 2016, 26 HOCM patients were treated at our hospital. All patients presented with palpitations, short breathing, or chest tightness after exercise. The
Table 1. Mitral Valve Conditions of the Patients

\begin{tabular}{lcc}
\hline Mitral Valve Conditions & Number & Percentage \\
\hline Mitral regurgitation & 6 & \\
$\quad$ Mild & 10 & 38.08 \\
$\quad$ Moderate & 10 & 38.46 \\
$\quad$ Severe & & \\
Pathology & 2 & 50.00 \\
Leaflet elongation & 2 & 50.00 \\
Leaflet thickening & & \\
$\quad$ Treatment & & \\
None & 22 & 84.62 \\
Mitral valvuloplasty & 4 & 15.38 \\
Mitral valve repair & 0 & 0.00
\end{tabular}

symptoms were not controlled by calcium channel blockers or beta-blockers. All methods were performed in accordance with the relevant guidelines and regulations. Information about informed written consent and approval for the study is in the Acknowledgments.

Echocardiography and magnetic resonance imaging (MRI) showed asymmetric hypertrophy of the left ventricular wall and septum, systolic anterior motion of the mitral apparatus (Figure 1), and marked obstruction of the left ventricular outflow tract. The mean left ventricular outflow tract gradient was greater than $50 \mathrm{~mm} \mathrm{Hg}$. All patients were in New York Heart Association (NYHA) class III-IV.

All surgeries were performed with general anesthesia and hypothermic extracorporeal circulation. Extracorporeal circulation was established by intubating the ascending aorta, superior vena cava, and inferior vena cava. After cardiac arrest, an incision was made obliquely or transversely at the aortic root. The right coronary cusp was retracted to thoroughly explore the hypertrophied septum and the anterior mitral leaflet. The upper borderline of septal myectomy was $3 \mathrm{~mm}$ inferior to the right coronary cusp and the aortic annulus. The right borderline of septal myectomy was the midpoint of the right coronary sinus. Resection was carried out left by 10-12 $\mathrm{mm}$ to the contact of left and right coronary cusps and was carried out inferiorly by $40-50 \mathrm{~mm}$ to the base of the mitral papillary muscles, including the abnormal muscles in the apex that fuse with the septum. The depth of septal myectomy reached $50 \%$ of the septal base. The mitral papillary muscles were mobilized from the ventricular walls to release the adhesion. In comparison with the classic Morrow septal myectomy, the modified Morrow procedure extends the resection area beyond the membranous part of the septum (Figure 2) [Song 2016].

Continuous data were presented as mean \pm standard deviation. The $t$ test was performed to compare the means in different groups. The statistical analyses were performed by using IBM SPSS Statistics for Windows, Version 21.0 (IBM Corp., Armonk, NY, USA; released 2012). $P<.05$ was considered statistically significant. 
Table 2. The Echocardiography Results*

\begin{tabular}{lcccc}
\hline Ultrasonic Indicator & Baseline & Postoperative 1 Week & P1 & Postoperative 1 Year \\
\hline Left ventricular outflow tract gradient & $93.36 \pm 7.93$ & $16.73 \pm 2.02$ & $<.001$ & $16.98 \pm 2.33$ \\
Left ventricular outflow tract velocity & $472.40 \pm 22.12$ & $188.40 \pm 14.16$ & $<.001$ & $189.33 \pm 14.23$ \\
Septal thickness & $19.43 \pm 0.77$ & $16.45 \pm 0.76$ & $<.001$ & $16.55 \pm 0.83$ \\
Mitral regurgitation area & $8.41 \pm 0.69$ & $3.04 \pm 0.73$ & $<.001$ & .000 \\
Left ventricular end-diastolic diameter & $44.71 \pm 0.99$ & $40.38 \pm 1.60$ & $<.001$ & $4.95 \pm 0.66$ \\
Left ventricular end-systolic diameter & $28.10 \pm 0.90$ & $26.24 \pm 1.18 \pm 1.36$ & .000 \\
Ejection fraction & $66.48 \pm 1.53$ & $61.44 \pm 2.65$ & $<.001$ & .000 \\
NYHA class & III-IV & $1-11$ & .001 & .000 \\
\hline
\end{tabular}

*P1, postoperative 1 week versus baseline; P2, postoperative 1 year versus baseline; NYHA, New York Heart Association.

\section{RESULTS}

The baseline characteristics were summarized in Table 1. The preoperative mitral regurgitation was mild in 6 patients, moderate in 10 patients, and severe in 10 patients. Marked mitral valve thickening was seen in 2 patients, and mitral leaflet elongation was seen in 2 patients. One patient had concomitant coronary atherosclerotic heart disease, one patient had paroxysmal atrial fibrillation, one patient had aortic regurgitation, and one patient had previous alcohol septal ablation (Table 1). Concomitant mitral valvuloplasty was performed in 4 patients, coronary artery bypass grafting was performed in one patient, and aortic valve replacement was performed in one patient. No in-hospital death occurred during our study. No interventricular septum perforation or complete atrioventricular block was noticed.

In comparison with baseline, the left ventricular outflow tract gradient was significantly reduced at postoperative 1 week $(16.73 \pm 2.02$ versus $93.36 \pm 7.93, P<.001)$ and 1 year $(16.98 \pm 2.33$ versus $93.36 \pm 7.93, P<.001)$. Septal thickness was significantly reduced at postoperative 1 week $(16.45 \pm$ 0.76 versus $19.43 \pm 0.77, P<.001)$ and 1 year $(16.55 \pm 0.83$ versus $19.43 \pm 0.77, P<.001)$ in comparison with baseline. Mitral regurgitation area was significantly decreased at postoperative 1 week $(3.04 \pm 0.73$ versus $8.41 \pm 0.69, P<.001)$ and 1 year $(2.95 \pm 0.66$ versus $8.41 \pm 0.69, P<.001)$ in comparison with baseline. Left ventricular outflow tract velocity, left ventricular end-diastolic diameter, left ventricular end-systolic diameter, and ejection fraction showed the same trend at postoperative 1 week and 1 year (Table 2 ). At postoperative 1 year, all patients were asymptomatic with significantly improved quality of life. The NYHA class was I-II for all patients. No reoperation was needed for any patients.

\section{DISCUSSION}

HOCM is also known as idiopathic hypertrophic subaortic stenosis, which is characterized by asymmetric hypertrophy of the muscular septum projecting into the left ventricular cavity. Systolic anterior motion of the mitral apparatus is frequent in HOCM, leading to obstruction of the left ventricular outflow tract, reduced left ventricular volume, and impaired diastolic function of the left ventricle. In HOCM patients, high flow velocity in the left ventricular outflow tract results in the Venturi effect [Mcintosh 1992], which causes anterior motion of the anterior mitral leaflet. The unequal lengths of the 2 mitral leaflets prevent the anterior motion of the posterior leaflet similar to that of the anterior leaflet, resulting in left ventricular outflow tract obstruction and a gap between the mitral leaflets. This finally results in systolic anterior motion of the mitral apparatus and mitral regurgitation deviated to the posterior junction during the mid-to-end systolic phase.

Septal myectomy is the standard treatment for HOCM [Mcintosh 1992; Yu 2000; Maron 2007]. This procedure can effectively reduce the septal thickness and the left ventricular outflow tract gradient, thus abolishing left ventricular outflow tract obstruction and reducing the risk of sudden cardiac death. Septal myectomy is indicated for symptomatic HOCM patients if the left ventricular outflow tract gradient is $\geq 50 \mathrm{~mm} \mathrm{Hg}$ at rest or $\geq 100 \mathrm{~mm} \mathrm{Hg}$ at provocation. Surgical treatment is also considered for young patients or children with mild symptoms but a left ventricular outflow tract gradient of 75-100 mm Hg at rest. However, some HOCM patients with concomitant submitral structure abnormalities treated with the classic Morrow procedure had persistent anterior motion of the mitral apparatus, leading to mitral regurgitation and left ventricular outflow tract obstruction [Jiang 1987; Minakata 2004].

In our study, $26 \mathrm{HOCM}$ patients were treated with the modified Morrow procedure for expanded myectomy. The postoperative mitral regurgitation and systolic anterior motion of the mitral apparatus were completely abolished or significantly relieved. Only one patient had moderate mitral regurgitation of $7 \mathrm{~cm}^{2}$ after the surgery. No surgeryrelated death occurred. At postoperative 1 year, all patients were asymptomatic, and the quality of life was significantly improved. The NYHA class was I-II. Echocardiography showed good anatomy and function of the mitral valve. No death, complications, or reoperation were recorded. 
The severity of mitral regurgitation is closely associated with the left ventricular outflow tract gradient, as well as the length and mobility of the mitral leaflets, and the inequality between the 2 mitral leaflets [Schwammenthal 1998; Minakata 2005]. For most patients, septal myectomy alone is adequate to abolish the mitral regurgitation and the systolic anterior motion of the mitral apparatus, and concomitant mitral valve surgery is unnecessary. A small proportion of patients still had mild mitral regurgitation and systolic anterior motion of the mitral apparatus after septal myectomy. The reasons include inadequate resecting of the hypertrophied myocardium, inadequate resecting of the middle septum, and not reaching the root of the mitral papillary muscles. This may result in postoperative left ventricular outflow tract obstruction, and reoperation is needed for severe cases [Klues 1992; Zhu 1992]. In addition, residual mitral regurgitation in a small number of patients after septal myectomy may be explained by focal mitral leaflet thickening or mild mitral annulus calcification.

Congenital abnormalities or secondary lesions in the mitral valve are found in approximately $10 \%-20 \%$ of the HOCM patients. For these patients, persistent mitral regurgitation and obstruction of the opening of the mitral valve may be noticed despite adequate septal myectomy and expansion of the left ventricular outflow tract. Various abnormalities of the mitral leaflets or submitral structures may be indicated by using echocardiography; these include mitral valve prolapse, chordal rupture, leaflet fibrosis, leaflet calcification, papillary muscle abnormality, rheumatic lesions, and infections. The adjacent mitral chordae may be incidentally injured during septal myectomy. In this case, mitral regurgitation-mostly deviated to the anterior junction or central part-can persist postoperatively even with a well-expanded left ventricular outflow tract, decreased left ventricular tract gradient, and abolished systolic anterior motion of the mitral apparatus The persistent mitral regurgitation has no obvious correlation with the left ventricular outflow tract gradient and the systolic anterior motion of the mitral apparatus. For this condition, concomitant mitral valvuloplasty or mitral valve repair is indicated. The goal of mitral valvuloplasty is to reduce the mitral leaflet area and correct the abnormal mitral apparatus. If needed, a larger-sized artificial annulus is preferred to avoid or minimize the risk of systolic anterior motion of the mitral apparatus [Kofflard 1996].

\section{LIMITATION}

Our study retrospectively analyzed the pathological characteristics of HOCM with concomitant mitral valve abnormalities and discussed the surgical treatment strategies. Despite the small number of cases and the lack of control group, all our patients achieved good anatomical and functional recovery.

\section{CONCLUSION}

In conclusion, our experience suggests that the modified Morrow myectomy with expanded resection area can effectively reduce the left ventricular outflow tract gradient and abolish the systolic anterior motion of the mitral apparatus. Mitral regurgitation associated with the obstruction is not surgically managed unless there are concomitant mitral valve lesions.

\section{ACKNOWLEDGMENTS}

The authors acknowledge the support and input from Beijing Anzhen Hospital, Capital Medical University.

\section{Funding: None.}

Ethics approval and consent to participate: All methods were performed in accordance with the relevant guidelines and regulations. Informed written consent was obtained from all participants before the enrollment in the study. Approval for the study was obtained from the Research Ethics Committee of Beijing Anzhen Hospital, Capital Medical University.

Competing interests: The authors declare that they have no competing interests.

Authors' contributions: B.R.S. performed or participated in the study and wrote the article. Y.L.R. interpreted the patient data. H.J.Z. revised the article. All authors read and approved the final article.

\section{REFERENCES}

Elliott P, McKenna WJ. 2004. Hypertrophic cardiomyopathy, Lancet 363(9424):1881-91.

Gersh BJ, Maron BJ, Bonow RO, et al. 2011. 2011 ACCF/AHA guideline for the diagnosis and treatment of hypertrophic cardiomyopathy: executive summary: a report of the American College of Cardiology Foundation/American Heart Association Task Force on Practice Guidelines J Thorac Cardiovasc Surg 142(6):1303-38.

Jiang L, Levine RA, King ME, Weyman AE. 1987. An integrated mechanism for systolic anterior motion of the mitral valve in hypertrophic cardiomyopathy based on echocardiographic observations. Am Heart J 113(3):633-44.

Klues HG, Maron BJ, Dollar AL, Roberts WC. 1992. Diversity of structural mitral valve alterations in hypertrophic cardiomyopathy. Circulation 85(5):1651-60.

Kofflard MJ, van Herwerden LA, Waldstein DJ, et al. 1996. Initial results of combined anterior mitral leaflet extension and myectomy in patients with obstructive hypertrophic cardiomyopathy. J Am Coll Cardiol 28(1):197-202.

Maron BJ. 2002. Hypertrophic cardiomyopathy: a systematic review. JAMA 287(10):1308-20.

Maron BJ. 2007. Surgical myectomy remains the primary treatment option for severely symptomatic patients with obstructive hypertrophic cardiomyopathy. Circulation 116(2):196-206.

Maron MS, Olivotto I, Betocchi S, et al. 2003. Effect of left ventricular outflow tract obstruction on clinical outcome in hypertrophic cardiomyopathy. N Engl J Med 348(4):295-303.

McIntosh CL, Maron BJ, Cannon RO 3rd, Klues HG. 1992. Initial results of combined anterior mitral leaflet plication and ventricular septal myotomy-myectomy for relief of left ventricular outflow tract obstruction in patients with hypertrophic cardiomyopathy. Circulation 86(5 suppl):II60-7.

Minakata K, Dearani JA, Nishimura RA, Maron BJ, Danielson GK. 2004. Extended septal myectomy for hypertrophic obstructive cardiomyopathy with anomalous mitral papillary muscles or chordae. J Thorac Cardiovasc Surg 127(2):481-9. 
Minakata K, Dearani JA, Schaff HV, O'Leary PW, Ommen SR, Danielson GK. 2005. Mechanisms for recurrent left ventricular outflow tract obstruction after septal myectomy for obstructive hypertrophic cardiomyopathy. Ann Thorac Surg 80(3):851-6.

Schwammenthal E, Nakatani S, He S, Hopmeyer J, et al. 1998. Mechanism of mitral regurgitation in hypertrophic cardiomyopathy: mismatch of posterior to anterior leaflet length and mobility. Circulation 98(9):856-65

Song B, Ran D. 2016. Comparison of modified with classic Morrow septal myectomy in treating hypertrophic obstructive cardiomyopathy. Medicine (Baltimore) Jan;95(2):e2326.

Yu EH, Omran AS, Wigle ED, Williams WG, Siu SC, Rakowski H. 2000. Mitral regurgitation in hypertrophic obstructive cardiomyopathy: relationship to obstruction and relief with myectomy. J Am Coll Cardiol 36(7):2219-25.

Zhu WX, Oh JK, Kopecky SL, Schaff HV, Tajik AJ. 1992. Mitral regurgitation due to ruptured chordae tendineae in patients with hypertrophic obstructive cardiomyopathy. J Am Coll Cardiol 20(1):242-7. 